\title{
Vascular Network Analysis with Manual and Automatic Segmentation Using Fuzzy Mathematical Morphology
}

\author{
Pedro Bibiloni $^{a, b}$ and Manuel González-Hidalgo ${ }^{a, b}$ and Arnau Mir ${ }^{a, b}$ and Nuria Prats-Domínguez ${ }^{a}$ \\ ${ }^{a}$ Department of Mathematics and Computer Science, SCOPIA Research Group, \\ University of the Balearic islands, 07122 Palma, Spain \\ \{p.bibiloni, manuel.gonzalez, arnau.mir\}@uib.es, nuria.prats10@gmail.com \\ ${ }^{b}$ Balearic Islands Health Research Institute (IdISBa), 07010 Palma, Spain
}

\begin{abstract}
The inhibition of new vessel creation, or anti-angiogenesis, has therapeutic interest. In this contribution, we introduce an automatic method to estimate objective indicators of new vessel creation. We use images of the chorioallantoic membrane of chicken embryos, some of which have been injected with a varying degree of methyl jasmonate, which has anti-angiogenic effects. We use fuzzy mathematical morphology to compute an automatic vessel segmentation, which along with the manual ground truth is used to study which features indicating the creation of new vessels can be replicated. Employing hypothesis tests and a PCA analysis, we conclude that our limited database provides positive but moderate results in terms of the statistical significance of the difference of such samples.
\end{abstract}

Keywords: Image processing, Vessel segmentation, Fuzzy Mathematical Morphology, Vascular features, Angiogenesis, Choriallantoic membrane

\section{Introduction}

Artificial vision, or computer vision, is the discipline that studies how to automatically process, analyze and interpret images. It includes the ability of computers to analyze images captured by a camera and obtain information of the objects present in the scene.

Automation is particularly useful in fields in which there is a vast amount of information. For instance, given the large number of digital images acquired, automation may be of great help in tasks comprising biomedical images, satellite images, security images, etc. Generally, biomedical images are interpreted by human experts. However, in some cases, the development of image analysis algorithms may automate many tasks, becoming thus a great tool for the human analyst. For instance, if the conditions of the images are not good, or there are a large number of them, the work of analyzing them becomes extremely tedious and monotonous, being prone to mistakes. Also, the development of quantitative measures makes the estimation of some biomarkers less subjective.

Angiogenesis is a biological process that regulates the formation of new capillaries from pre-existing vessels in the body through a branching process [3]. It is considered essential in several physiological processes, such as tissue growth and remodeling. Stimulating the growth of new blood vessels has been proposed as a therapeutic approach to treat diseases including ischemic heart disease - which is the main cause of mortality in the world-, neurodegenerative disorders or hair loss [5]. On the other hand, angiogenesis is not always beneficial. It is believed that an excessive and unregulated angiogenic response contributes to diseases such as rheumatoid arthritis, psoriasis, tumor growth and metastasis, diabetic retinopathy and macular degeneration related to age. For this and for therapeutic reasons, it is also interesting to study how to inhibit the formation of new blood vessels, also known as antiangiogenesis [14].

Generally, the study of angiogenesis is based on the study of microscopic images. It is usually carried out with visual examinations aimed at characterizing the vascular network, including manually counting features, manually extracting global measures, and manually quantifying the changes observed. Due to the growth of the set of images to be processed, their manual inspection becomes an extremely laborious and inefficient task with a high degree of subjectivity, whose results are very difficult to reproduce [4]. Thus, the design and implementation of image-processing methods, which are reproducible, has become one of the main goals in the quantification of angiogenesis. 
The main problem with the investigation of angiogenesis is the choice of adequate trials to evaluate the efficacy of new potential drugs [16]. Classic trials to study angiogenesis in vivo are based on the bag of the hamster cheek; the ears, the skin and the dorsal aerial sack of the rabbit; the Chorioallantoic Membrane (CAM) of chicken embryos; and the iris and the avascular cornea of the eye of rodents. One of the most popular, on which we will base our work, is the one based on the CAM, which has certain advantages such as its low cost, simplicity and reliability [14]. To access the CAM there are two main methods: one is to allow the embryo to develop and then extract it through a window in the shell, and the other is to cultivate the embryo in a petri dish, without the shell. Finally, once the CAM has been accessed, images of it are taken, which are later used to quantify the angiogenesis.

So far all the studies related to the quantification of angiogenesis begin by processing images of the vascular network to estimate the binary mask of blood vessels, from which global measures are calculated. The automation of such process would be a great advance, as well as the calculation of the local measurements - describing each individual bifurcation.

The vast majority of approaches involving fuzzy techniques and soft computing relate to the segmentation of the vascularity. Kande et al. used fuzzy $c$-means to segment vascularity in fundus images [7]. Bibiloni et al. explored the use of fuzzy mathematical morphology to segment vessels as curvilinear objects [2] Forkert et al. used a voxel-wise fuzzy approach to fuse information as a preprocessing step before vessel segmentation [6].

Objectively measuring the vascularity on digital images has become a topic widely studied in recent years. Nikiforidis et al. studied the vessels in CAM images, which they categorize based on their diameter [10]. They extracted features based on these categories, specifically the vascular area and the total length of vessels in each category. Wild et al. manually estimated the number of vessels with a digital imageassisted method, which includes manual thresholding and morphological skeleton [18]. Other authors presented automatic methods, without any manual stage involved. Li et al. automatized the measurement of angiogenesis in 3D images of cerebral microcirculatory networks of mice, obtained with Optical Coherence Angiography (OCTA) [8]. After automatically segmenting the vascular network, they measure the amount of vascularity based on the density of pixels of the skeletonized vascular network. Doukas et al. used CAM images to extract measures based on an automatic binarization of the image converted to grayscale [5]. They extracted several automatic measures, such as vessel total length, vascular density and branching points, as well as texture-based features. Delgado also used CAM images to extract a wider range of measures, including ratios between vessels in bifurcations and bifurcation angles [4].

In this work, we introduce and study a method to automatically quantify the angiogenesis using CAM images. The aim of this work is twofold. First, to introduce a method to automatically detect the presence of angiogenesis. That is, to design and implement imageprocessing algorithms and mathematical models that are capable of reproducing the characteristics that a human expert would extract from the aforementioned CAM images and their corresponding expert segmentation. These characteristics include the number of terminal points, the number of bifurcation points, the density of the vascular region, and the density of the vascular size, among others. Second, to obtain an automatic segmentation of the CAM images and to estimate the characteristics from such segmentation, with the aim of comparing them to those obtained with the ground truth.

\section{Materials and methods}

In this section, we introduce the experimental settings. In particular, we describe the images of the dataset used, a high-level description of the features of interest that we aim at estimating and the automatic vessel segmentation algorithm.

\subsection{Dataset}

We use images of the Chorioallantoic Membrane (CAM) of chicken embryos, taken with a Nikon Stereoscopic Zoom Microscope SMZ-1800 connected to a digital camera DS-U1 Nikon. The zoom used was $2 \times$, and the resolution of the images is $1280 \times 960$ pixels. In the Computer Department of the Faculty of Mathematics and Computation of the Universidad de Oriente, in conjunction with the Department of Physics and Pharmacy of the University of Sao Paulo, Brazil, researchers are developing a project to quantify the Walker's tumor-induced angiogenesis in CAM images obtained with the aforementioned Stereoscopic Microscope [1]. To our knowledge, this is the first dataset with digitalized images of the vascular network and the vascular network manually segmented by a specialist, the vascular Ground Truth (GT). A detailed description of the preparation of the samples and the image capture settings can be found in $[4,11]$.

The dataset contains 30 CAM samples, being either control samples or samples treated with methyl jasmonate (MJ), a compound whose anti-angiogenic effects on various experimental environments have been 


\begin{tabular}{lccccc} 
& $0 \mu \mathrm{L}$ & $25 \mu \mathrm{L}$ & $50 \mu \mathrm{L}$ & $100 \mu \mathrm{L}$ & Total \\
\hline Control & 4 & & & & 4 \\
W-MJ & & 5 & 4 & 2 & 11 \\
L-Control & & & & 5 & 5 \\
L-MJ & & 3 & 5 & 2 & 10 \\
\hline
\end{tabular}

Table 1: Number of images in the dataset according to the injected substance, where $\mathrm{W}$ and $\mathrm{L}$ correspond to the suspension solvents, water and liposome; and Control and MJ indicate the absence or presence of methyl jasmonate.

reported in the literature [11]. The samples are further split into samples treated with or without a liposome suspension, which can act as a carrier of MJ. Table 1 shows a summary of the samples according to their treatment. In particular, 11 CAM samples were treated with the following doses of MJ: 25, 50 or 100 $\mu \mathrm{L}$ in water suspension (second row of Table 1 ); and 10 CAM samples were treated with the same doses of MJ in a liposome suspension (fourth row of Table 1). In addition, the first group of 11 CAM samples is matched with 4 control samples (first row of Table 1), with no injection; whereas the liposome suspension group is matched with 5 control samples (third row of Table 1).

In Figure 1, we show two images of the dataset. As we can observe, the images shown present uneven illumination. In general, microscopic image capturing is often affected by intensity variations. This phenomenon is known as shading inhomogeneity [17]. It must be corrected before an automatic image processing analysis.

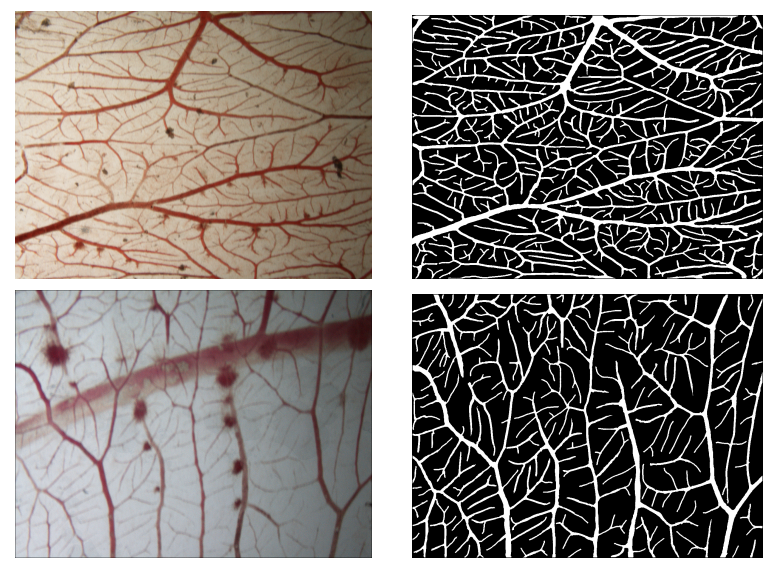

Figure 1: Original CAM images (left) and vessel GT segmentation provided by the specialist (right).

\section{$2.2 \quad$ Feature extraction}

By reviewing the literature on the subject, we selected the set of characteristics that may help to discriminate the presence or absence of angiogenesis $[5,8,10,11$, $15,18,19]$.

We emphasize that, in this first work, we only consider the features that are employed by human experts when they manually classify the samples. In particular, we have not considered features based on texture, fractal dimension, or other based on non-specialized image processing techniques. Also, we classify them into two groups: global features and local features, being the latter measures taken on each vessel bifurcation and then averaged across the whole image. The extracted features are described in the following.

\section{Global features}

Taking into account the entire image of the blood vessels, the following measures can be estimated:

Terminal points (TP). Number of points located at the end of each blood vessel. A higher number of terminal points often reflects the proliferation of blood vessels.

Bifurcation points (BP). Number of points from which a blood vessel splits into two or more vessels. It it increases, it can also indicate a higher proliferation of blood vessels.

Density of vascular area (DVA). Ratio between the area of the vascular network with respect to the total area of the image. That is, DVA $=\frac{\mathrm{VA}}{\mathrm{TA}}$, where VA is the total number of pixels belonging to vessels (white pixels in the GT image), and TA is the total area of the image. In our CAM images, $\mathrm{TA}=1280 \times 800$ pixels. A more intense angiogenic process could reflect a higher density of vascular area.

Density of vascular network (DVN). Each vessel of the vascular network can be associated to its central line. The set of all these lines constitutes the skeleton of the vascular network. The density of the vascular network is defined as the ratio between the length of this skeleton and the total area of the image. That is, $\mathbf{D V N}=\frac{\mathrm{SL}}{\mathrm{TA}}$, where SL is the skeleton total length, in pixels.

The computation of the feature DVA is straightforward, since we always estimate them from a binary mask of the vessels, in white, on a black background. The algorithms to estimate the TP, BP and the skeleton to compute the DVN, all of them based on the 


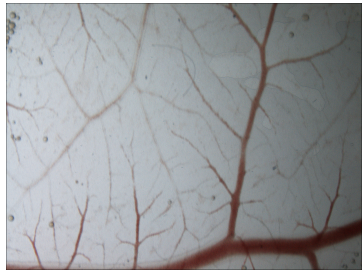

(a) Original image.

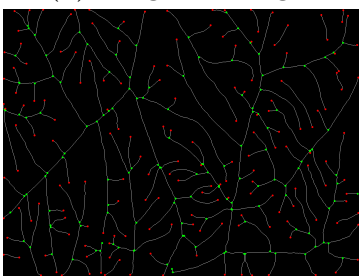

(b) Vessel segmentation

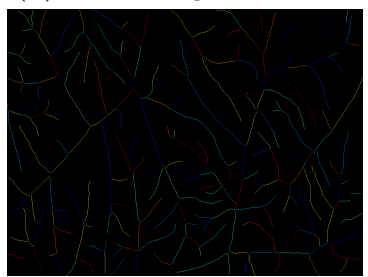

(c) Skeleton with terminal (d) Connected components points in red and the bifur- highlighted in different colcation points in green. ors.

Figure 2: Detection of terminal points, bifurcation points and the connected components of the skeleton, eliminating the bifurcation points and an 8neighborhood of each one of the bifurcation points.

morphological analysis of binary images, have been implemented in Matlab. To do so, we first obtain the skeleton of the vessels segmentation image by refining the results of the morphological thinning in order to avoid little branches and double bifurcation points. After that, we obtain the terminal points and the bifurcation points with the morphological operations of endpoints and branch-points, respectively, with a postprocessing refinement to merge close points. The results of this image analysis procedure are ordered lists of the terminal points, the bifurcation points and the connected components of the skeleton. See Figure 2 for an example where we show the results of this analysis on a CAM image.

\section{Local features}

Focusing now on each set of vessels connected by a bifurcation point, we have considered the following local measures, which also characterize the structure of the vascular network [15] and, therefore, the changes in this structure that could indicate the presence or absence of angiogenesis.

Roy and Woldenberg describe the elements of a vascular bifurcation [15]. The bifurcation exponent establishes a relationship between the diameter of a parent vessel, $d_{0}$, and the diameter of its daughter vessels, $d_{1}$ and $d_{2}$, with $d_{0}>d_{1} \geq d_{2}$ (see Figure 3 ) which is as follows:

$$
d_{0}^{x}=d_{1}^{x}+d_{2}^{x} .
$$

There is no general solution for determining the value of the exponent $x$, and different alternatives have been postulated. Murray proposed, in [9], that $x=3$ based on the assumption that, for laminar flow, the flowradius relationship is given by

$$
f=k r^{3},
$$

equation that was derived by minimizing the total power loss involved in the transport and maintenance of the blood volume within the vessel. However, the Murray model has been generalized [15], specifically to situations where the flow-radius relationship is given by $f=k r^{x}$, with $x$ ranging between 2 and 3 depending on the underlying assumptions. In this work, following $[9,15]$, we consider the estimation $x=3$.
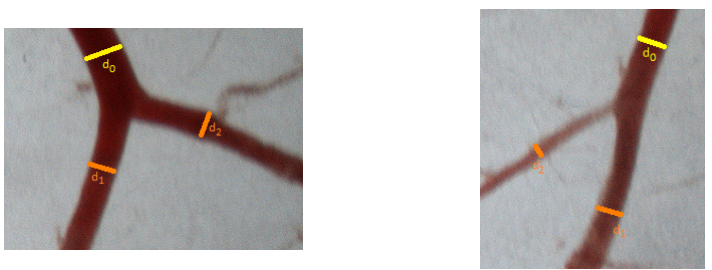

Figure 3: Vessel bifurcation with diameters marked: in yellow, the diameter of the main vessel; in orange, the diameter of the daughter vessels.

Taking into account each of the set of vessels connected by the same bifurcation point, the following characteristics are estimated.

Bifurcation index (BI). It relates the width between two blood vessels with the same ascendant [15]. That is,

$$
\gamma=\frac{d_{2}}{d_{1}}
$$

where $d_{1} \geq d_{2}$. It reflects the symmetry of the bifurcation.
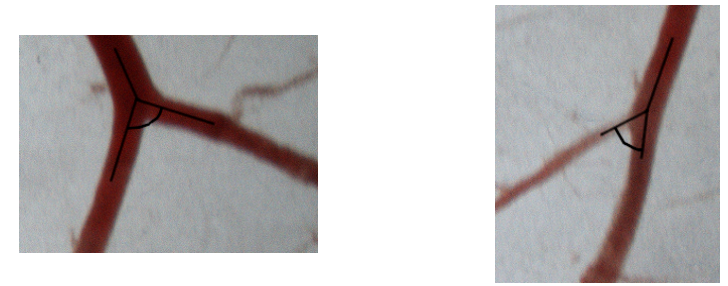

Figure 4: Vessels bifurcation with the bifurcation angle marked in black.

Bifurcation angle (BA). The bifurcation of blood vessels results in a bifurcation angle, between the descendant vessels, which indicates how the vascular network is expanding. Under the assumption of minimum volume and minimum power 
loss, the bifurcation angle, $\psi$, can be computed as [15]:

$$
\cos \psi=\frac{\left(1+\gamma^{x}\right)^{2-4 / x}-\gamma^{2 x-4}-1}{2 \gamma^{x-2}},
$$

or, alternatively, as:

$$
\cos \psi=\frac{d_{0}^{4}-d_{1}^{4}-d_{2}^{4}}{2 d_{1}^{2} d_{2}^{2}} .
$$

Since the number of bifurcation points is considerably high, the points that do not meet the relation (1) are not taken into account when we compute the bifurcation angle using the previous equations. That is, they are discarded for this specific feature under the basis that they do not meet the underlying assumptions.

Diameter relation (DR). As the name suggests, it is a measure that relates the diameters of the different blood vessels connected. In contrast to the bifurcation index, the ratios of interest are those of the main vessel with respect to each of its daughter vessels [15].

$$
\begin{gathered}
\frac{d_{1}}{d_{0}}=\left(1+\left(\frac{d_{2}}{d_{1}}\right)^{x}\right)^{-\frac{1}{x}}=\frac{1}{\left(1+\gamma^{x}\right)^{\frac{1}{x}}}, \\
\frac{d_{2}}{d_{0}}=\frac{d_{2}}{d_{1}}\left(1+\left(\frac{d_{2}}{d_{1}}\right)^{x}\right)^{-\frac{1}{x}}=\frac{\gamma}{\left(1+\gamma^{x}\right)^{\frac{1}{x}}} .
\end{gathered}
$$

Area ratio (AR). Relation between the width of the parent vessel and the combined width of its daughter vessels. It measures the expansion or contraction of the cross sectional area at each bifurcation. The area of proportion, $\beta$, is defined as:

$$
\beta=\frac{d_{1}^{2}+d_{2}^{2}}{d_{0}^{2}}
$$

This coefficient can also be expressed in terms of the bifurcation index, $\gamma$, and of the bifurcation exponent, $x$, being rewritten as follows [15]:

$$
\beta=\frac{1+\gamma^{2}}{\left(1+\gamma^{x}\right)^{\frac{2}{x}}} .
$$

\subsection{Fuzzy mathematical morphology}

To obtain an automatic segmentation of the vascular network we employ an algorithm based on the fuzzy mathematical morphology. It is briefly introduced here for the sake of completeness, more information can be found in [2]. The fuzzy mathematical morphology is a comprehensive extension of the binary morphology in which the fuzzy logic operators fuse the information of the neighbourhood indicated by the template known as structuring element. Since fuzzy logic operators typically admit elements within the range $[0,1]$, we will assume that the pixels of our grayscale images also have such range. The values of the structuring element, similarly encoded as a grayscale image, also range in $[0,1]$.

The fuzzy mathematical morphology is based on two operators, the fuzzy dilation and the fuzzy erosion. The former enlarges bright objects, whereas the latter shrinks them. More formally, let us consider an image $A$ and a structuring element $B$. Then, given a fuzzy implication function $I$ and a fuzzy conjunction $C$, we define the fuzzy dilation of $A$ by $B, \mathcal{D}_{C}(A, B)$; and the fuzzy erosion of $A$ by $B, \mathcal{E}_{I}(A, B)$, as:

$$
\begin{aligned}
& \mathcal{D}_{C}(A, B)(y)=\sup _{x} C(B(x-y), A(x)), \\
& \mathcal{E}_{I}(A, B)(y)=\inf _{x} I(B(x-y), A(x)) .
\end{aligned}
$$

Combining them and denoting $\bar{B}(x)=B(-x)$, we define the fuzzy black top-hat transform as:

$$
\mathcal{B} \mathcal{T} \mathcal{H}_{C, I}(A, B)=\mathcal{E}_{I}\left(\mathcal{D}_{C}(A, B), \bar{B}\right)-A \text {. }
$$

This transform highlights the regions that can not be recovered by the erosion after the dilation. That is, the thin and dark regions that are completely removed by the dilation, which in our case correspond precisely to the vessels.

\subsection{Segmentation algorithm}

The vessel segmentation algorithm that we have used in this work is based on the fuzzy black top-hat detector described in [2]. The different stages of the algorithm are:

Preprocessing. Firstly, a shading correction method is applied, to achieve the maximum possible homogenization of the illumination of the CAM images. A homomorphic filter is used, see [17]. Secondly, the RGB image is transformed to the CIELab color space, whose $L$ channel is contrasted with the CLAHE algorithm [12] as enhancement step, with $16 \times 16$ tiles.

Fuzzy black-top hat detector. The detector based on the fuzzy black top-hat transformation described in [2] is used on the image obtained in the preprocessing step. As mentioned above, the goal of this step is to enhance the thin regions that are darker than their neighbouring regions. With it, we obtain a fuzzy map of the degrees with which each pixel belongs to a vessel. In particular, we use the minimum conjunction, $C(x, y)=\min (x, y)$, and its residuated implication, the Gödel implication $I(x, y)=\mathbb{1}_{x \leq y}+y \cdot \mathbb{1}_{x>y}$.

Binarization. Then, a hysteresis algorithm is applied to binarize the image while the connected components 
tend to be retained. The lower and higher thresholds have been experimentally set to, respectively, the percentiles 0.060 and 0.105 of the maximum minus the minimum.

Postprocessing. Finally, we remove small regions and fill small holes in the binarized images. Specifically, we remove the 8-connected components with less than 15 pixels, and we fill the 4-connected holes with less than 15 pixels. This last step provides the final vessel estimation.

In Figure 5, we can observe the final vessel estimation obtained by the previous algorithm applied to he original CAM image. In terms of quantitative measures comparing this segmentation with its corresponding GT [2], we obtain a precision of 0.6919 , a sensitivity of 0.7036 , a specificity of 0.8959 , and an F1-score of 0.6977. Mean quantitative measures for all the images can be found in Table 3 .
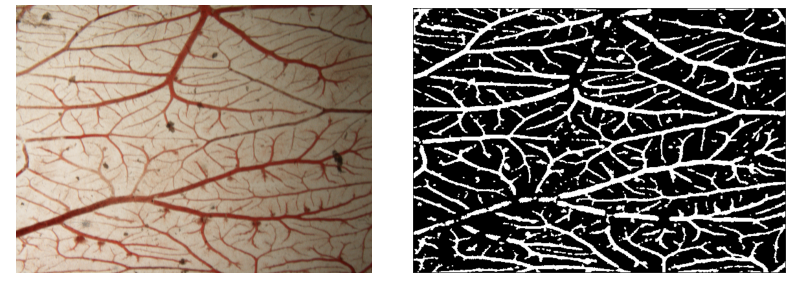

Figure 5: Original CAM image (left), and automatic vessel segmentation (right), of a sample whose GT is displayed in Figure 1.

\section{Numerical experiments and analysis}

We recall that, in this work, we aim at studying three main hypothesis:

- First, verify the manual analysis of the vascular study by comparing the samples treated with methyl jasmonate, and the control samples.

- Second, find out, based on the selected features and the vessels manually extracted, whether one can distinguish the treatment of each sample.

- Third, validate the segmentation method in terms of quantitative metrics between the automatic segmentation and the GT.

To carry out the first task, a hypothesis test has been performed, for each feature, to check whether the mean obtained with affected samples is significantly different to the mean obtained with control samples. In particular, we used the t-test if the two samples passed a Shapiro-Wilk normality test, or the Wilcoxon signedrank test otherwise. We emphasize that the hypothesis

\begin{tabular}{|c|c|c|c|c|c|}
\hline & & \multicolumn{2}{|c|}{ Water susp. } & \multicolumn{2}{|c|}{ Liposome susp. } \\
\hline & & $25 \mu \mathrm{L}$ & $50 \mu \mathrm{L}$ & $25 \mu \mathrm{L}$ & $50 \mu \mathrm{L}$ \\
\hline \multirow{2}{*}{$\mathrm{TP}$} & $p$-value & 0.265 & 0.105 & 1 & 0.122 \\
\hline & Stat. diff. & $x$ & $x$ & $x$ & $x$ \\
\hline \multirow{2}{*}{$\mathrm{BP}$} & $p$-value & 0.243 & 0.082 & 0.014 & 0.167 \\
\hline & Stat. diff. & $x$ & $x$ & $\checkmark$ & $x$ \\
\hline \multirow{2}{*}{ DVA } & $p$-value & 0.466 & 0.237 & 0.112 & 0.688 \\
\hline & Stat. diff. & $x$ & $x$ & $x$ & $x$ \\
\hline \multirow{2}{*}{ DVN } & $p$-value & 1 & 0.342 & 0.030 & 0.215 \\
\hline & Stat. diff. & $x$ & $x$ & $\checkmark$ & $x$ \\
\hline \multirow{2}{*}{$\begin{array}{l}\text { BI } \\
\text { (mean) }\end{array}$} & $p$-value & 0.926 & 0.363 & 0.010 & 0.030 \\
\hline & Stat. diff. & $x$ & $x$ & $\checkmark$ & $\checkmark$ \\
\hline \multirow{2}{*}{$\begin{array}{l}\text { BA } \\
(\text { mean })\end{array}$} & $p$-value & 1 & 1 & 0.351 & 0.470 \\
\hline & Stat. diff. & $x$ & $x$ & $x$ & $x$ \\
\hline \multirow{2}{*}{$\begin{array}{l}\text { DR } \\
\text { (mean) }\end{array}$} & $p$-value & 0.422 & 0.693 & 1 & 0.125 \\
\hline & Stat. diff. & $x$ & $x$ & $x$ & $x$ \\
\hline \multirow{2}{*}{$\begin{array}{l}\text { DR } \\
\text { (mean) }\end{array}$} & $p$-value & 0.592 & 1 & 0.671 & 0.073 \\
\hline & Stat. diff. & $x$ & $x$ & $x$ & $x$ \\
\hline
\end{tabular}

Table 2: Statistical study to check whether the features computed from the treated samples and from control samples provide significantly different estimators, with $\alpha=0.05$. We emphasize that this does not reflect the predictive power of each feature, but whether their automatic estimation is statistically indistinguishable to their corresponding expert ground truth.

tests have been carried out for the categories in which at least 3 samples were available (see Table 1), and that we used a significance level of $\alpha=0.05$.

Table 2 shows the results of these comparisons, where we observe that very few features reflect a significance difference. In particular, we found significant differences in the following cases: the number of bifurcation points for samples injected with $25 \mu \mathrm{L}$ of L-MJ; the density of vascular network for samples with $25 \mu \mathrm{L}$ of L-MJ; and the mean of bifurcation indices of samples with 25 and $50 \mu \mathrm{L}$ of L-MJ. For the rest of the variables, we conclude that there is no significant difference between the treated and the control samples.

We have faced the second task with a Principal Components Analysis (PCA). The two most informative principal components have been calculated, separately for the features computed from the manual GT and for the features based on the automatic segmentation algorithm. For both groups of images, the features with the most influence in this analysis of principal components have been the number of terminal points, the number of bifurcation points and the mean of the cosinus of the bifurcation angles.

The results of this analysis can be seen in Fig- 
ure 6. The samples injected with water suspension are coloured in red, and their control samples, in blue. The samples injected with liposome suspension are coloured in green, and their control samples in black. In both cases, the two principal components explain more than the $98 \%$ of the variability of all the features. As it can be seen, in the two cases, the control samples are difficult to separate from the samples with substance. Uniquely, the samples treated with a liposome suspension seem to be moderately separable from their corresponding control samples.

The results corresponding to the third objective are shown in Table 3. Specifically, we have computed the precision, sensitivity, specificity and $F_{1}$-score $[13,2]$. The value of the specificity, or true negative rate, is high, which indicates that the segmentation method is able to detect those pixels that do not belong to the segmentation. However, the sensitivity, or true positive rate, is relatively low. This indicates that the segmentation could be improved by better detecting positive pixels. Finally, the $F_{1}$-score, which is the harmonic mean between the precision and the sensitivity, is comparable to values obtained in other vascular segmentation settings [2].

\begin{tabular}{cccc}
\hline Precision & Sensitivity & Specificity & $F_{1 \text {-score }}$ \\
\hline 0.7071 & 0.4554 & 0.9600 & 0.5382 \\
\hline
\end{tabular}

Table 3: Mean of the quantitative metrics to compare automatic vessel segmentations with their corresponding manual GT.

\section{Conclusions}

We have presented a contribution to the analysis of vascular networks using manual and automatic segmentation. In particular, we study the anti-angiogenic effect of the methyl jasmonate injected either in a water suspension or in a liposome suspension. We hypothesize that such injection may introduce changes in the vessels captured by specific metrics. To verify this, we have performed several statistical tests to see if the mean of the features of the injected samples are different from the mean of the control samples. However, only the number of bifurcation points, the density of vascular area and the density of vascular network have been proved to be significantly different in the case of 25 and $50 \mu \mathrm{L}$ of a liposome suspension with MJ.

Also, a PCA was conducted to check whether it is possible to distinguish the sample images from the control images according to their features. Such analysis shows that all the groups are hardly separable. Since there is very few separability in the manual segmentation, the low separability in the manual segmenta- tion can not validate the method. However, to double check its correctness, some quantitative metrics have been calculated. The results show that the method has, in mean, high specificity, precision and $F_{1}$-score, while the sensitivity is moderate.

The results obtained differ significantly. First, although some features reflect the difference between samples in different groups, this difference can not be generalized to other features that are being used by human experts. We attribute this effect to the high demand that a statistical test represents and the low number of samples (see Table 1). Second, we consider that the automatic segmentation obtained with fuzzy mathematical morphology can be validated in terms of estimating the features of interest in this contribution. In particular, both vascular images presents approximately the same separability in terms of the PCA analysis, as well as having successful performance metrics. However, our segmentation presents different characteristics, such as the number of connected components, which could affect the estimation of high-level features.

\section{Acknowledgments}

This work was partially supported by the project TIN 2016-75404-P AEI/FEDER, UE.

\section{References}

[1] A. Alves, R. C. Guedes, L. V. Costa-Lotufo, et al., Modelo experimental de tumor na cavidade oral de ratos com carcinossarcoma de walker 256, Acta Cir Bras 19 (4) (2004) 354-60.

[2] P. Bibiloni, M. González-Hidalgo, S. Massanet, General-purpose curvilinear object detection with fuzzy mathematical morphology, Applied Soft Computing 60 (2017) 655-669.

[3] M.-L. Boizeau, P. Fons, L. Cousseins, et al., Automated image analysis of in vitro angiogenesis assay, Journal of Laboratory Automation 18 (5) (2013) 411-415.

[4] W. E. Delgado Font, Extracción de características para la cuantificación de la angiogénesis a partir de imágenes de la membrana corioalantoica de embriones de pollos, in: XIV Exposición Nacional Forjadores del Futuro, Cuba, 2014, pp. 1-18.

[5] C. N. Doukas, I. Maglogiannis, A. Chatziioannou, A. Papapetropoulos, Automated angiogenesis quantification through advanced image processing techniques, in: 2006 International Conference of the IEEE Engineering in Medicine and Biology Society, IEEE, 2006, pp. 2345-2348. 

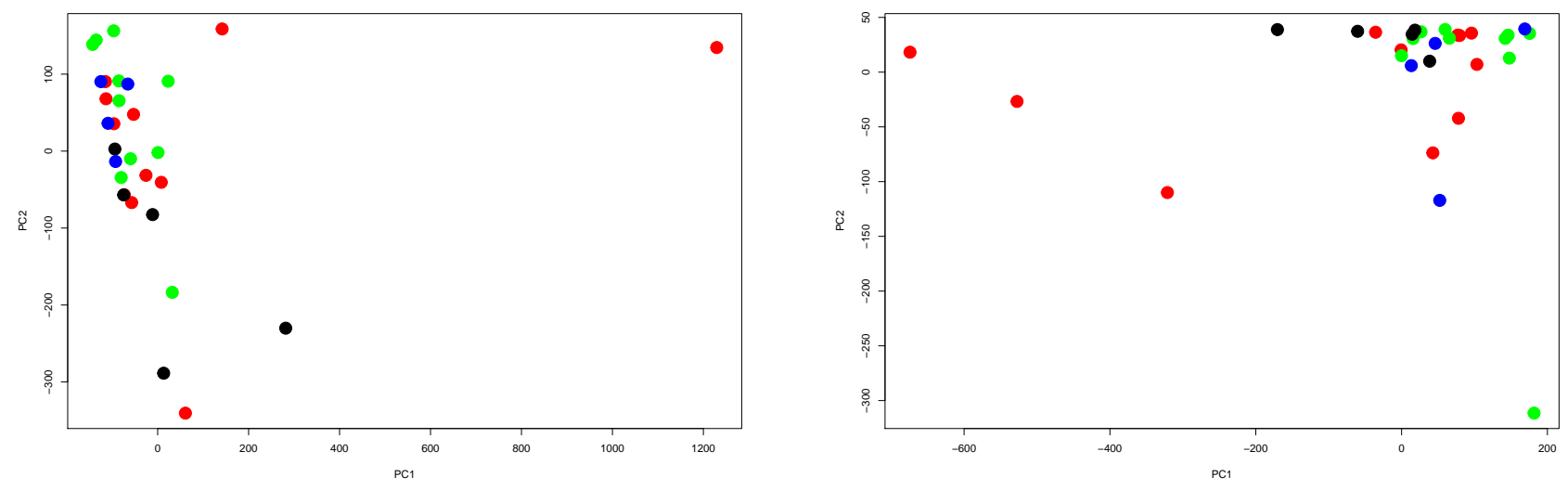

Figure 6: The two principal components using the manual vascular segmentation (left) and using the automatic vascular segmentation (right). In red, the samples with water suspension; in green, the samples with liposome suspension with MJ (WC); in blue, the control samples; and in black, the samples with liposome suspension without MJ.

[6] N. Forkert, A. Schmidt-Richberg, J. Fiehler, T. Illies, D. Möller, H. Handels, D. Säring, Fuzzybased vascular structure enhancement in timeof-flight mra images for improved segmentation, Methods of information in medicine 50 (01) (2011) 74-83.

[7] G. B. Kande, P. V. Subbaiah, T. S. Savithri, Unsupervised fuzzy based vessel segmentation in pathological digital fundus images, Journal of medical systems 34 (5) (2010) 849-858.

[8] A. Li, J. You, C. Du, Y. Pan, Automated segmentation and quantification of OCT angiography for tracking angiogenesis progression, Biomedical Optics Express 8 (12) (2017) 5604-5616.

[9] B. Moreau, B. Mauroy, Murray's law revisited: Quémada's fluids and fractal trees, Journal of Rheology 59 (6) (2015) 1419-1430.

[10] G. Nikiforidis, D. Papazafiropoulos, D. Siablis, et al., Quantitative assessment of angiogenesis in the chick embryo and its chorioallantoic membrane by computerised analysis of angiographic images, European Journal of Radiology 29 (2) (1999) 168-179.

[11] J. Pereira Lopes, M. Barbosa, C. Stella, W. Santos, et al., In vivo anti-angiogenic effects further support the promise of the antineoplasic activity of methyl jasmonate, Brazilian Journal of Biology 70 (2) (2010) 443-449.

[12] S. M. Pizer, E. P. Amburn, J. D. Austin, et al., Adaptive histogram equalization and its variations, Computer vision, Graphics, and Image Processing 39 (3) (1987) 355-368.
[13] D. M. Powers, Evaluation: From precision, recall and F-measure to ROC, informedness, markedness and correlation, Journal of Machine Learning Technologies 2 (1) (2011) 37-63.

[14] D. Ribatti, A. Vacca, L. Roncali, F. Dammacco, The chick embryo chorioallantoic membrane as a model for in vivo research on anti-angiogenesis, Current Pharmaceutical Biotechnology 1 (1) (2000) 73-80.

[15] A. G. Roy, M. J. Woldenberg, A generalization of the optimal models of arterial branching, Bulletin of Mathematical Biology 44 (3) (1982) 349-360.

[16] C. A. Staton, M. R. Reed, N. J. Brown, A critical analysis of current in vitro and in vivo angiogenesis assays, International Journal of Experimental Pathology 90 (2009) 195-221.

[17] D. Tomaževič, B. Likar, F. Pernuš, Comparative evaluation of retrospective shading correction methods, Journal of Microscopy 208 (3) (2002) $212-223$

[18] R. Wild, S. Ramakrishnan, J. Sedgewick, A. Griffioen, Quantitative assessment of angiogenesis and tumor vessel architecture by computerassisted digital image analysis: effects of VEGFtoxin conjugate on tumor microvessel density, Microvascular Research 59 (3) (2000) 368-376.

[19] B. Zhang, A. Liya, S. Li, Z. Xu, AngioIQ: A novel automated analysis approach for angiogenesis image quantification, in: 2nd International Conference on Biomedical Engineering and Informatics, IEEE, 2009, pp. 1-5. 